Short reports

\title{
The use of liver grafts infested with Clonorchis sinensis for orthotopic liver transplantation
}

\author{
CK Yeung, JKS Ho, WY Lau, KH Lee, AKC Li
}

\begin{abstract}
Summary
In the Orient, millions are known to have Clonorchis sinensis (biliary trematodiasis) infestation. When these infested livers become available as donor organs, there are potentially serious implications that the transplant team would need to consider. We report the use of two such infested livers, one from a cadaveric donor and the other from a live related donor, for orthotopic liver transplantation. The parasite was encountered not only during organ procurement, but also caused early postoperative blockage of the hepaticojejunostomy splintage tube and cessation of bile flow in the second recipient. Upon follow-up for four and two years, respectively, no other ill effect has been observed in either patient. Repeated examination of stool for ova of $C$ sinensis in both patients during follow-up visits had been negative, indicating that all the parasites probably had been killed by the cold perfusion. In view of the severe shortage of liver grafts and the lack of serious morbidity associated with the use of these infested livers, we have adopted the policy to include these livers for future transplantation. Special considerations and precautions are, however, required during the perioperative period and on long-term followup.
\end{abstract}

Keywords: Clonorchis sinensis, liver transplantation.

The highly successful results of orthotopic liver transplantation have revolutionized the management of end-stage liver disease in both adults and children. ${ }^{1,2}$ In most liver transplantation centres worldwide, however, organ shortage still poses a difficult problem. In the Orient, where eating raw fish is a common habit, millions of people are known to have Clonorchis sinensis (biliary trematodiasis) infestation. While the majority remain asymptomatic, when these individuals become potential liver donors (either cadaveric or living related), there may be important sequellae following the use of their infested livers for transplantation. We reported earlier the successful use of a cadaveric liver graft infested with $C$ sinensis for orthotopic liver transplantation in an adult with no ill effects. ${ }^{3}$ In this present report a reducedsize liver graft from a living donor infested with the parasite was used for transplantation in a small girl. Mechanical obstruction of the hepatico-jejunostomy splintage tube by dead carcasses of $C$ sinensis, which led to a cessation of bile flow, was encountered during the early postoperative period. The implications of this hitherto unreported complication as well as other possible long term morbidity associated with the use of livers infested with $C$ sinensis for liver transplantation need to be explored.

\section{Case reports}

\section{Case 1}

A 14-month-old, $9.2 \mathrm{~kg}$ girl with biliary atresia and after Kasai portoenterostomy was accepted as a candidate for liver transplantation. Because of extreme shortage of cadaveric donors and the patient's deteriorating condition, a living related transplantation became necessary. Her 41-year-old father, who had enjoyed good health previously, was evaluated. Endoscopic retrograde cholangiopancreatography (ERCP) was performed which showed a normal pancreatic duct although the bile duct could not be cannulated. However, intraoperative cholangiography was normal. Left hepatectomy with removal of segments II, III and IV was performed in standard fashion. When the segmental biliary duct was severed, two live $C$ sinensis were seen flushing out with bile. The donor liver was quickly cooled and perfused with University of Wisconsin (UW) solution. The perfused left lobe was further reduced to segments II and III. Throughout the bench work, no further $C$ sinensis was encountered. The donor liver was implanted with standard techniques. A 6 Fr Cook's tube was used as splintage tube for the hepaticojejunostomy. The patient recovered well from the procedure. At the 16th postoperative hour, there was cessation of bile flow via the splintage tube. This was initially observed, and with no improvement, the Cook's tube was irrigated at the 23rd hour, resulting only in 'dilute bile' at 1-2 $\mathrm{ml} / \mathrm{h}$. At the 26 th postoperative hour, the Cook's tube was forcefully flushed and irrigated. The culprit blockage from a dead $C$ sinensis was removed, with return of normal bile flow. Subsequent ultrasound studies and cholangiogram revealed patency of vessels and biliary tract. The patient was discharged from the hospital well and with normal liver function two months after the transplantation. Repeated stool examination during follow-up visits for ova of $C$ sinensis remained negative. In view of this and the stable liver function, prophylactic antihelminthic chemotherapy was not given. 
Features of Clonorchis sinensis infection

- mild $C$ sinensis infection is usually asymptomatic

- with heavier infestations, upper abdominal discomfort, diarrhoea and malaise may occur

- obstructive jaundice, hepatomegaly and recurrent pyogenic cholangitis can occur with very heavy infections

Case 2

A 60-year-old woman with cryptogenic cirrhosis resulting in end-stage liver failure underwent orthotopic liver transplantation using a cadaveric donor liver infested with $C$ sinensis. ${ }^{3}$ Dead carcasses of the parasite were encountered during bench work as well as during the implantation procedure. The patient recovered well after the operation. Repeated examination of the stool for ova of $C$ sinensis during followup visits was negative.

\section{Discussion}

Clonorchis sinensis (oriental liver flukes) are the commonest non-blood flukes endemic in most Southeast Asian countries where raw fish are consumed. Seah reported an infestation rate of $C$ sinensis at $15.5 \%$ among Chinese immigrants to the US, Canada, and other countries. ${ }^{4}$ Although heavy infestation with $C$ sinensis can cause various hepatobiliary disorders, in general most infested individuals are asymptomatic. Mild infestation with $C$ sinensis, therefore, are usually either unsuspected or undetected by conventional clinical evaluation. Owing to the high prevalence of infestation among people in the Southeast Asian countries, one would surmise that $C$ sinensis is likely to be encountered during organ harvesting and subsequent implantation of the allograft in liver transplantation.

In our first patient, we did find live $C$ sinensis towards the end of harvesting in the live donor when the biliary tract was opened. In both patients, however, only dead carcasses of the parasite were encountered after cold perfusion of the liver grafts, suggesting that the parasites

1 McMaster P, Dousset B. The improved results of liver transplantation. Transplant Int 1992; 5: 125-8.

2 Busuttil RW, Seu P, Millis J, et al. Liver transplantation in children. Ann Surg 1991; 213: 48-57.

3 Ho JK, Lau WY, Liu K, et al. Liver infested with Clonorchis sinensis in orthotopic liver transplantation: a case report. Transplant Proc 1994; 26: 2269-71.

\section{Learning points}

- the use of liver grafts infested with $C$ sinensis is a feasible option and should be considered by transplant teams, especially in the Orient

- prophylactic antihelminthic chemotherapy is not necessary posttransplant as all the parasites are probably killed during the cold preservation procedures

- biliary blockage by dead $C$ sinensis causing cessation of bile flow may be encountered during the early postoperative period

might have been killed by the cold preservation procedures. In our first patient, there was early cessation of bile flow from the biliary splintage tube due to blockage by the dead parasites. This had lasted for 10 hours before the culprit $C$ sinensis was successfully removed by forceful flushing and aspiration. We therefore need to consider biliary blockage by dead $C$ sinensis as a possible cause of diminution in bile flow during the early postoperative period. Failure to recognize this previously unreported complication may lead to unnecessary removal of the splintage tube and therefore avoidable morbidity. Theoretically, an unyielding blockage by heavy loads of carcasses of $C$ sinensis can cause potentially serious biliary complication which may require re-exploration. For this reason, it is still conjectural whether one should consider discarding donor livers which are heavily infested by $C$ sinensis. Should such donor livers be used, either no stent or a large size splintage tube is recommended for biliary reconstruction.

With the influx of Asian immigrants from originally endemic areas of $C$ sinensis infestation to many western countries, liver transplant teams worldwide will need to be aware of this problem. Recipients of infested donor livers will need to be monitored for eggs of $C$ sinensis in the stools and treated accordingly with signs of clinical infection. As the mucin-secreting type of cholangiocarcinoma is associated with $C$ sinensis in Hong Kong, ${ }^{5}$ and the risk of development of cholangiocarcinoma with prolonged immunosuppression after transplantation with an infested donor liver is still unknown, life-long surveillance for development of malignancy will be needed.

4 Seah SKK. Intestinal parasites in Chinese immigrants in Canadian city. F Trop Med Hyg 1973; 76: 291.

5 Hon PC. The relationship between primary carcinoma of the liver and infestation with Clonorchis sinensis. F Pathol Bacteriol 1956; 72: 239. 